\title{
LIGHT-INDUCED DEFECTS IN THERMAL ANNEALED HYDROGENATED AMORPHOUS SILICON
}

J. Serra, J. Bertomeu, G. Sardin, C. Roch, J.M. Asensi, J. Andreu*, and J.L. Morenza. University of Barcelona, Department of Applied Physics and Electronics Avda. Diagonal 647, E-08028 Barcelona, Spain.

\begin{abstract}
The metastable defects of a-Si:H samples annealed at temperatures in the $300-550^{\circ} \mathrm{C}$ range have been studied by photothermal deflection spectroscopy (PDS). The light-soaked samples show an increase in optical absorption in the 0.8 to $1.5 \mathrm{eV}$ range. The metastable defect density decreases when the annealing temperature increases, while the defect density increases. This decrease in the metastable defect density shows an almost linear correlation with the decrease in the hydrogen content of the samples, determined by IR transmission spectroscopy and thermal desorption spectroscopy.
\end{abstract}

${ }^{*}$ To whom the correspondence should be addressed. 


\section{Introduction}

Hydrogenated amorphous silicon (a-Si:H) is a semiconductor that is widely used in photovoltaic applications. In addition to its low scale applications in consumer products, the middle scale in remote applications is now at an advanced stage of industrial development. The creation of light-induced defects is one of the relevant problems which is the object of intense investigation. As a consequence of this effect, the efficiency of a-Si:H based solar cells decreases after the initial exposure to light. A better understanding of the creation of lightinduced defects and a technological optimization of the material in order to reduce them is necessary to increase the long-term performance of a-Si:H solar cells. Since the discovery of the phenomenon [1], much work has been done in order to understand the mechanisms involved.

It seems clear that the creation of metastable defects is produced by the recombination of electron-hole couples [2], or utmost by charge trapping [3]. It is also accepted that this effect, although influenced by the presence of high impurity concentrations [4], is intrinsic to hydrogenated amorphous silicon.

From the microscopical point of view, there are several models that attempt to explain the metastable behaviour. Some of the models appeal to an appreciable displacement of atoms, namely the models based on Si-Si bond breaking. The model of Stutzmann et al. [4] proposes the rupture of a weak Si-Si due to a neighbouring hydrogen bond, along with the creation of a dangling bond and a Si-H-Si center. Street and Winer [5] have presented thermodynamic models in order to study the thermal equilibration of dangling bonds with weak bonds and have tested four microscopic mechanisms with the experimental results. Pantelides [6] assumes the existence of overcoordinated silicon defects called floating bonds and has presented a model including both dangling bonds and floating bonds, without considering any influence of hydrogen in the light-induced metastable effect. Jackson [7] considers the dissociation of the $\mathrm{H}_{2}{ }^{*}$ complex into neutral hydrogen at a bond-centered site and neutral hydrogen at a tetrahedral site that diffuses fast until breaking a weak $\mathrm{Si}-\mathrm{Si}$ bond, two dangling bonds being created as 
a result of the whole process.

Several results point to a relevant role of hydrogen in the light-induced metastability. For example, Jackson [8] relates the hydrogen diffusion to the decay of light-induced defects, and Chen [9] observes appreciable changes in the density of metastable defects after prolonged exposition of the samples to atomic hydrogen. Moreover Ohsawa [10] observes a decrease in the metastable defect density measured by ESR in samples annealed at temperatures in the $300-450^{\circ} \mathrm{C}$ range.

This latter result has attracted our attention and the purpose of the present work is to carry out a detailed investigation of annealed samples using photothermal deflection spectroscopy (PDS). 


\section{Experimental}

The experiment consists in the annealing of a-Si:H samples at temperatures between $300-550{ }^{\circ} \mathrm{C}$ and subsequent illumination for $16 \mathrm{~h}$. Both states, annealed and illuminated, are studied by PDS in order to obtain the defect density.

The samples were prepared by depositing $3 \mu \mathrm{m}$ of device-quality a-Si:H on Corning 7059 glass by RF (13.56 MHz) glow-discharge decomposition of pure silane. The RF power, the substrate temperature and the gas pressure were kept at their optimum values for our reactor $\left(5 \mathrm{~W}, 280^{\circ} \mathrm{C}, 33 \mathrm{~Pa}\right)$.

The samples were annealed under vacuum $\left(3 \times 10^{-5} \mathrm{~Pa}\right)$ for two hours at the temperatures indicated in Table I.

The hydrogen content of the samples was determined by infrared transmission spectroscopy, integrating the peak of Si-H wagging mode following Cardona [11]. These results are in good agreement with those obtained by thermal desorption spectroscopy [12].

A transverse and fully automatized PDS setup was used. From the PDS signal, the energy dependence of the absorption coefficient was obtained by means of the incoherent approximation [13]. In the region of the Urbach edge this dependence can be represented by $\alpha(h v)=10^{3} \exp \left[\left(h v-E^{03}\right) / E_{0}\right] \mathrm{cm}^{-1}$, where $E_{0}$ and $E^{03}$ characterize the width and position of the Urbach edge respectively. The subgap density of states $\left(\mathrm{N}_{\mathrm{S}}{ }^{0}\right)$ was obtained following Jackson [14].

The behaviour of the saturation value of the PDS signal was compared between the samples annealed at different temperatures. A slight increase in the saturation value was found in samples annealed at higher temperatures, which may have been due to an increase in the surface roughness and a consequent decrease in the reflection coefficient, or to the variation in optical gap when hydrogen is removed from the sample. No differences between the saturation values were observed between the sample before and after light soaking.

The accuracy in the measurement of the density of defects was tested in two ways. Firstly, we calculated the influence of the fitting of the position and width of Urbach edge on 
the integration of subgap absorption, using a cut-off value of $1.5 \mathrm{eV}$. In this case, a variation of $1 \%$ in the $\mathrm{E}_{0}$ value (due to a slightly different selection of the Urbach edge zone in which to carry out the fitting) induces a fluctuation of $2.5 \%$ in the calculated $\mathrm{N}_{\mathrm{S}}$ value. The second test is the fluctuation of subgap absorption values among different measurements of the same sample or among measurements of different samples. In this case the deviation of the $\mathrm{N}_{\mathrm{S}}$ values from their mean is $4.8 \%$, calculated as the integration of the standard deviation of the subgap absorption, so this fluctuation is independent of fitting of the Urbach edge. The fluctuation in the values of the Urbach edge width found in different samples was about $1 \%$, so they are in the range accepted as the correct value obtained from the fitting.

For PDS measurements the samples are dipped in $\mathrm{CCl}_{4}$ and we have observed that thermal annealing of samples exposed to $\mathrm{CCl}_{4}$ produces an increase in the sub-gap absorption, due to contamination. In order to avoid this disturbance, the measurements were carried out on different $0.5 \times 2 \mathrm{~cm}^{2}$ strips of the same sample, each one annealed at a fixed temperature. The reliability of this method is based in the reproducibility of the different strips of the sample, as discussed above. For the as-deposited sample, the corresponding values of $\mathrm{N}_{\mathrm{S}}$ and $\mathrm{E}_{0}$ are in the ranges $(1.36 \pm 0.08) \times 10^{16} \mathrm{~cm}^{-3}$ and $55.0 \pm 0.5 \mathrm{meV}$ respectively. These values are in line with those reported in the literature for device-quality material.

In order to study the creation of light-induced defects for the samples annealed at different temperatures, the subgap absorption was measured by PDS immediately after the annealing (state A) and after $16 \mathrm{~h}$ of illumination with unfiltered light from a SCHOTT KL 1500 lamp with ellipsoid halogen reflection bulb 15V/150W (state B). Further annealing of state $\mathrm{B}$ at $180{ }^{\circ} \mathrm{C}$ for $1.5 \mathrm{~h}$ leads to a similar PDS spectrum to that of the original state $\mathrm{A}$, as can be observed in Figure 1. The corresponding values of defect density of state A, state B and recovered state A are $1.36 \times 10^{16} \mathrm{~cm}^{-3}, 2.77 \times 10^{16} \mathrm{~cm}^{-3}$ and $1.40 \times 10^{16} \mathrm{~cm}^{-3}$ respectively. The number of defects produced by light soaking was determined from the difference in subgap absorption in states (A) and (B) as measured by PDS, instead of substracting the $\mathrm{N}_{\mathrm{S}}$ values of the two states, in order to reduce the possible error in $\mathrm{N}_{\mathrm{S}}$ calculation due to the Urbach edge fitting which would make the absolute error and the $\Delta \mathrm{N}_{\mathrm{S}}$ values comparable. 


\section{Results and discussion}

The absorption spectra corresponding to intrinsic a-Si:H annealed at different temperatures are shown in Figure 2. No significant change in the absorption spectra is observed at annealing temperatures below $350^{\circ} \mathrm{C}$. As the annealing temperature is raised to $400^{\circ} \mathrm{C}$, subgap absorption significantly increases, while the exponential absorption region broadens and shifts to lower energies. The values of the Urbach edge parameters $\mathrm{E}_{\mathrm{O}}$ and $\mathrm{E}^{\mathrm{O} 3}$ and the defect density $\mathrm{N}_{\mathrm{S}}{ }^{0}$ are indicated in Table I, and Figures 3 and 4 show their variation with the annealing temperature. In amorphous semiconductors, the Urbach edge width is coupled to the disorder of the lattice [15]. These results are thus interpreted as an increase in the disorder and the defect density associated with the hydrogen effusion.

The increase in the absorption coefficient $(\Delta \alpha)$ caused by illumination as a function of photon energy is shown in Figure 5. In order to verify the accuracy of $\Delta \alpha$ in these results, the standard deviation corresponding to five measurements of the absorption coefficient in an asdeposited sample, is also plotted. The results indicate that the light-induced absorption increment lowers when the annealing temperature increases. Assuming no changes in the matrix element for optical transitions, this behaviour may be interpreted as a decrease in the metastable state density induced by annealing. The integration of the $\Delta \alpha$ spectra up to $1.5 \mathrm{eV}$, where the error is already too high, gives the light-induced defect density $\Delta \mathrm{N}_{\mathrm{S}}$.

The values of defect density after light soaking $\mathrm{N}_{\mathrm{S}}{ }^{\mathrm{i}}$ and the light-induced increase in the defect density $\Delta \mathrm{N}_{\mathrm{S}}$ are shown in Table I, and they are plotted as a function of the annealing temperature in Figure 4. It can be seen that light-induced defects decrease from $1.32 \times 10^{16}$ to $1.0 \times 10^{15} \mathrm{~cm}^{-3}$ when the annealing temperature rises from $300^{\circ} \mathrm{C}$ to $500^{\circ} \mathrm{C}$. For higher temperatures the light-induced defects become undetectable in our experimental system. It is to be noted that the light-induced defect density decreases while the total defect density increases.

The hydrogen content of the annealed samples is shown in Table I, and Figure 6 represents its relation with the density of light-induced defects. A decrease in light-induced 
defects can be observed when the hydrogen content decreases.

The first important fact to be remarked concerns to the initial density of states of samples annealed at moderate temperatures (below $400^{\circ} \mathrm{C}$ ). Increases in the density of states with respect to the as-deposited sample of about $10^{15}$ are obtained when more than $10^{21}$ hydrogen atoms have evolved. It seems unlikely that so many hydrogen atoms could produce a similar increase in the number of tail states, and this fact can be easily interpreted by considering a reconstruction of the lattice and an increase in the extended states in the band. The proximity between three-coordinated $\mathrm{Si}$ atoms is required for this reconstruction, so hydrogen evolved at low temperatures probably comes from clustered hydrogen.

At higher annealing temperatures, when only about $2 \%$ of hydrogen remains in the sample, the ratio between created defects and evolved hydrogen increases. This behaviour could be interpreted assuming that the hydrogen evolved at the final step of effusion comes from isolated hydrogen atoms. The absence of other three-coordinated $\mathrm{Si}$ atoms in the neighbourhood disables the lattice reconstruction, which would involve the formation of new Si-Si bonds.

The density of photogenerated defects in the range of hydrogen concentrations between $16 \%$ and $2 \%$ decreases linearly (Figure 6). This can be interpreted by assuming that the clustered hydrogen is the center responsible for the defect creation mechanism. In this concentration range, the concentration of isolated hydrogen remains almost constant whereas the weak-bond density increases. So, models implying the weak-bond break as the only mechanism for the creation of metastable centers, without considering the role of hydrogen in this process, seem to be in disagreement with experimental results.

As a consequence, these results seem to be particularly in agreement with the model of Jackson [7], in which the $\mathrm{H}_{2}{ }^{*}$ complex is presented as the center responsible for the light-induced defect creation.

The present results of photogenerated defects are measured after only $16 \mathrm{~h}$ illumination, by which time the defect creation process has still not reached saturation. The present study focuses only on the behaviour in the initial stages of defect creation. This should be borne in 
mind when interpreting the results. Further measurements are required to elucidate the saturation situation.

The saturation studies require tedious experimental work, but can be quantitatively interpreted with thermodynamical models (such as the models proposed by Street and Winer [5]), extended to an electronic non-equilibrium situation. These models consider the equilibration between dangling bonds and weak bonds and give the equilibrium densities as a function of microscopical parameters. In order to compare the results, it would be necessary to evaluate the light-induced change of dangling-bond density under illumination. 


\section{Conclusions}

In conclusion, the results of the sub-gap optical absorption measurements indicate an increase in dangling bonds with light soaking. The moderate increase in the density of states in samples annealed at lower temperatures supports the hypothesis of lattice reconstruction after hydrogen removal. The metastable defect density of samples decreases when the annealing temperature increases, while the defect density increases. This decrease in the metastable defect density presents an almost linear correlation with the hydrogen content, which highlights the role of hydrogen in the metastability mechanisms.

\section{Acknowledgements}

This study was supported by DGICYT of the Spanish Government (project PB89-0236). One of the authors (J.B.) acknowledges partial support from CIRIT of the Generalitat de Catalunya (AR89). 


\section{References}

[1] D.L. Staebler and C.R. Wronski, Appl. Phys. Lett. 31 (1977) 292.

[2] R.A. Street, Appl. Phys. Lett. 41 (1982) 1060.

[3] A.R. Hepburn, J.M. Marshall, and C. Main, Phys. Rev. Lett. 56 (1986) 2215.

[4] M. Stutzmann, W.B. Jackson, and C.C. Tsai, Phys. Rev. B 32 (1985) 23.

[5] R.A. Street and K. Winer, Phys. Rev B 40 (1989) 6236.

[6] S.T. Pantelides, Phys. Rev. B 36 (1987) 3479.

[7] W.B. Jackson, Phys. Rev. B 41 (1990) 10257.

[8] W.B. Jackson and J. Kakalios, Phys. Rev. B 37 (1988) 1020.

[9] Y.A. Chen, Appl. Phys. Lett. 53 (1988) 1277.

[10] M. Ohsawa, T. Hawa, T. Ichimura, T. Akasaka, H. Sakai, S. Ishida, and Y. Uchida, J. Non-Cryst. Solids 77\&78 (1985) 401.

[11] M. Cardona, Phys. Stat. Sol. 118 (1983) 463.

[12] J. Serra, J. Andreu, G. Sardin, C. Roch, J.M. Asensi, J. Bertomeu, J. Esteve, Physica B 170 (1991) 269.

[13] N.M. Amer and W.B. Jackson, in Semiconductors and semimetals vol.21 "Hydrogenated amorphous silicon" part B, edited by J.I. Pankove. (Academic Press, 1984), p. 83 .

[14] W.B. Jackson and N. M. Amer, Phys. Rev. B 25 (1982) 5559.

[15] J. Singh, Phys. Rev. B 23 (1981) 4156. 


\section{Figure Captions}

Figure 1. PDS deduced absorption spectra of intrinsic a-Si:H at initial state $(\circ)$, after illumination for $16 \mathrm{~h}(\square)$ and after $1.5 \mathrm{~h}$ annealing at $180^{\circ} \mathrm{C}(\triangle)$.

Figure 2. PDS deduced absorption spectra of intrinsic a-Si:H, after annealing at different temperatures.

Figure 3. The width $E_{0}(\square)$ and position $E^{03}(\circ)$ of the Urbach edge, as a function of annealing temperature for intrinsic a-Si:H.

Figure 4. Defect density of the initial state $(O)$, after illumination $(\triangle)$, and light-induced defect density $(\bullet)$, as a function of the annealing temperature.

Figure 5. Absorption coefficient variation after illumination in samples annealed at different temperatures, (a) $300^{\circ} \mathrm{C}$, (b) $350^{\circ} \mathrm{C}$, (c) $400^{\circ} \mathrm{C}$, and the standard deviation corresponding to five measurements of the absorption coefficient in an as-deposited sample (d).

Figure 6. Light-induced defect density as a function of the hydrogen content after the annealings. 
Table I. Effects of annealing on the a-Si:H properties.

\begin{tabular}{|c|c|c|c|c|c|c|}
\hline $\begin{array}{c}\mathrm{T} \\
\left({ }^{\circ} \mathrm{C}\right)\end{array}$ & $\begin{array}{c}E_{0} \\
(\mathrm{meV})\end{array}$ & $\begin{array}{l}E^{3} \\
(\mathrm{eV})\end{array}$ & $\begin{array}{c}\mathrm{N}_{\mathrm{S}} \\
\left(10^{16} \mathrm{~cm}^{-3}\right)\end{array}$ & $\begin{array}{c}\mathrm{N}_{\mathrm{S}}^{i} \\
\left(10^{16} \mathrm{~cm}^{-3}\right)\end{array}$ & $\begin{array}{c}\Delta \mathrm{N}_{\mathrm{S}} \\
\left(10^{16} \mathrm{~cm}^{-3}\right)\end{array}$ & $\begin{array}{c}7 \text { Hecontent } \\
(\%)\end{array}$ \\
\hline $280^{+}$ & 55 & 1.724 & 1.36 & 2.68 & 1.32 & 15.3 \\
\hline 300 & 55 & 1.724 & 1.36 & 2.68 & 1.32 & 12.3 \\
\hline 325 & 55 & 1.717 & 1.68 & 2.30 & 0.62 & 9.3 \\
\hline 350 & 56 & 1.724 & 1.42 & 2.21 & 0.79 & 10.9 \\
\hline 375 & 59 & 1.712 & 2.15 & 2.93 & 0.78 & 7.1 \\
\hline 400 & 61 & 1.707 & 3.92 & 4.42 & 0.50 & 7.7 \\
\hline 450 & 63 & 1.700 & 5.27 & 5.37 & $0.1^{*}$ & 4.0 \\
\hline 500 & 64 & 1.675 & 4.54 & 4.65 & $0.1^{*}$ & 2.0 \\
\hline 550 & 70 & 1.593 & 10.4 & 10.4 & - & 0 \\
\hline
\end{tabular}

$(+)$ as deposited

(*) determined with high uncertainty 


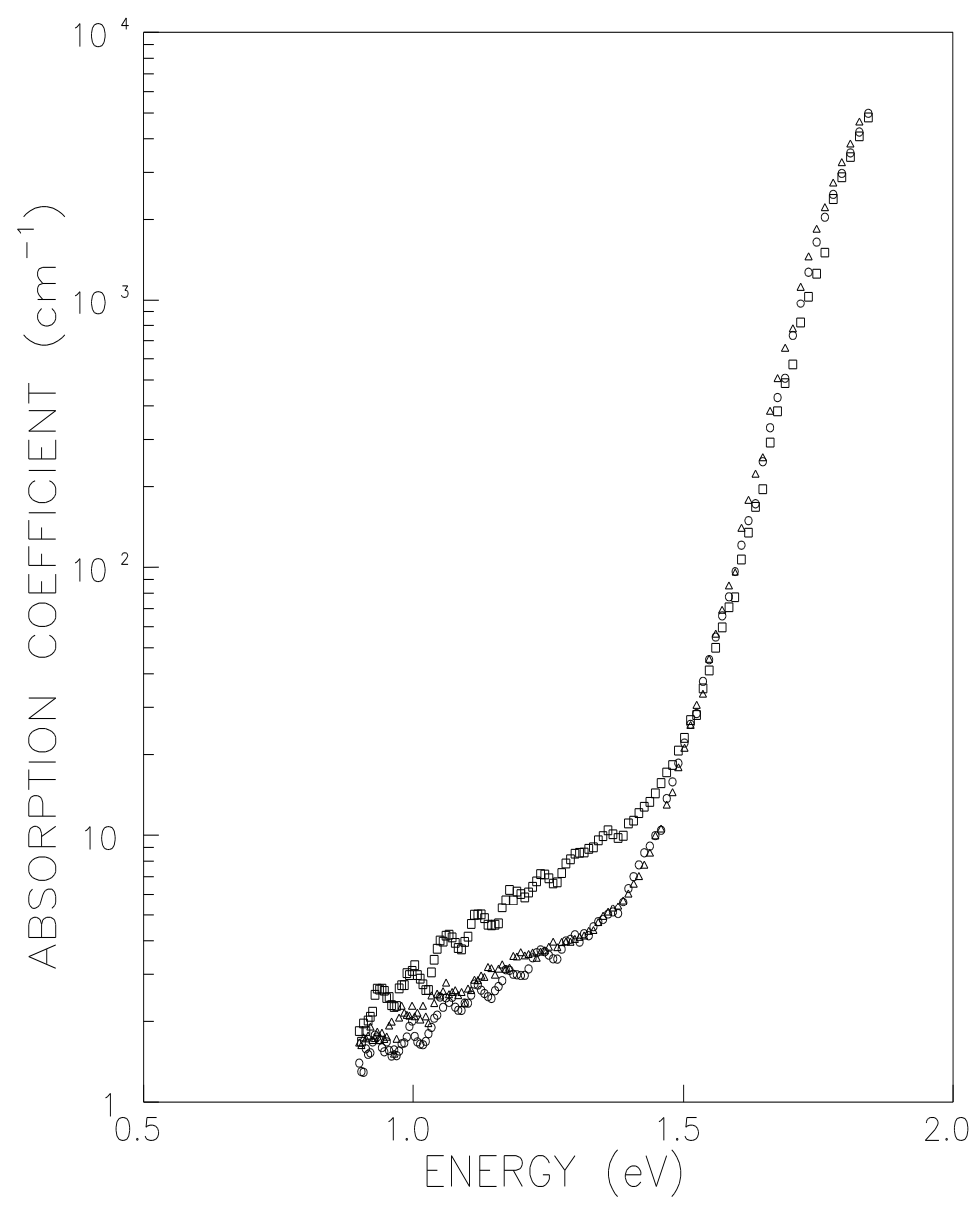




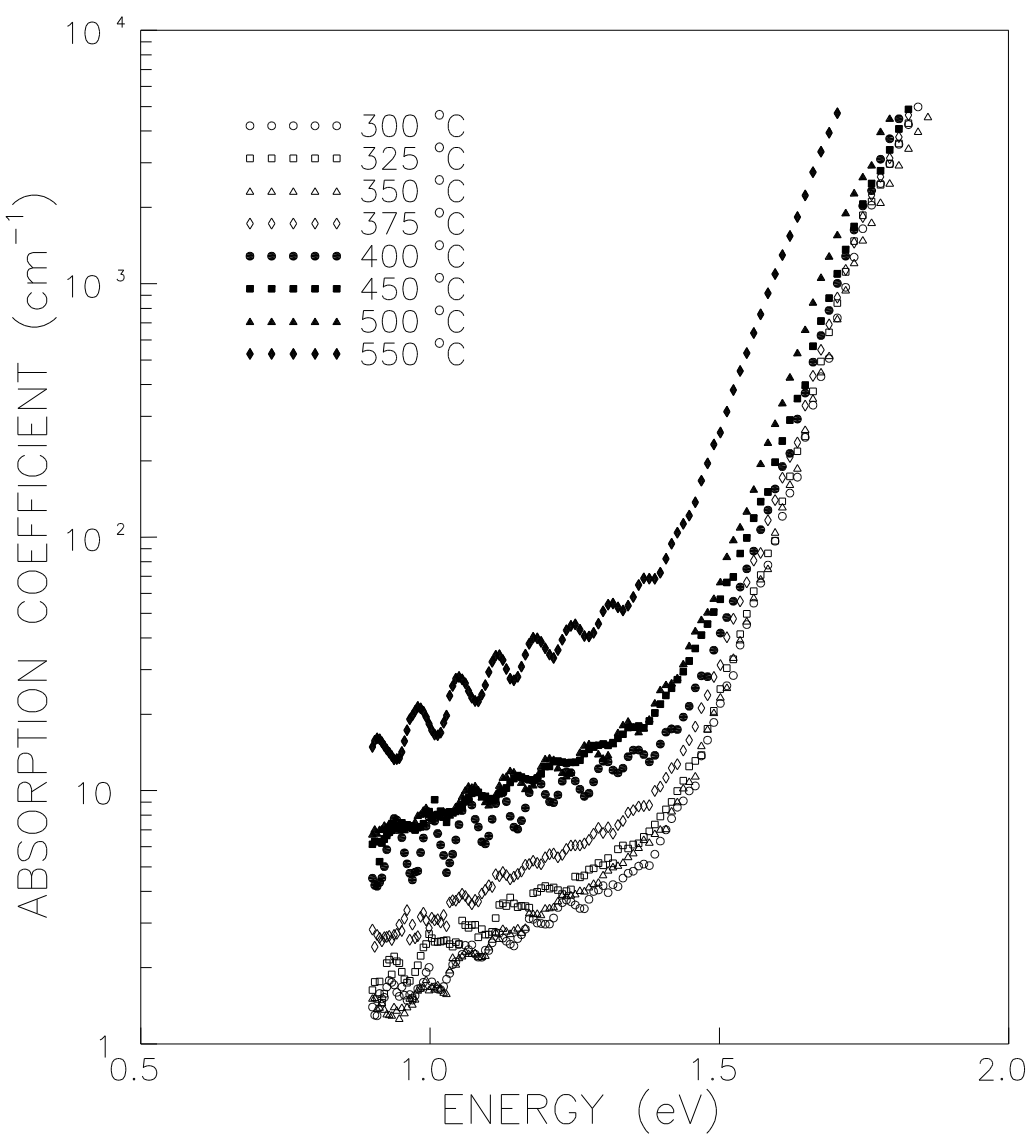




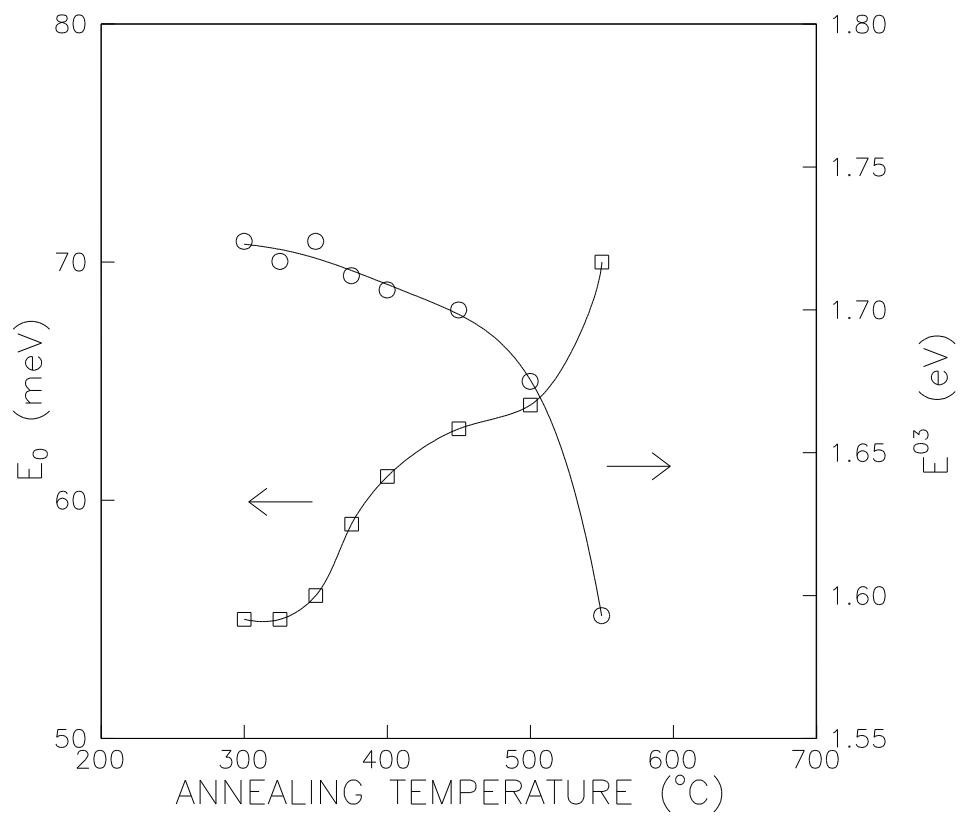




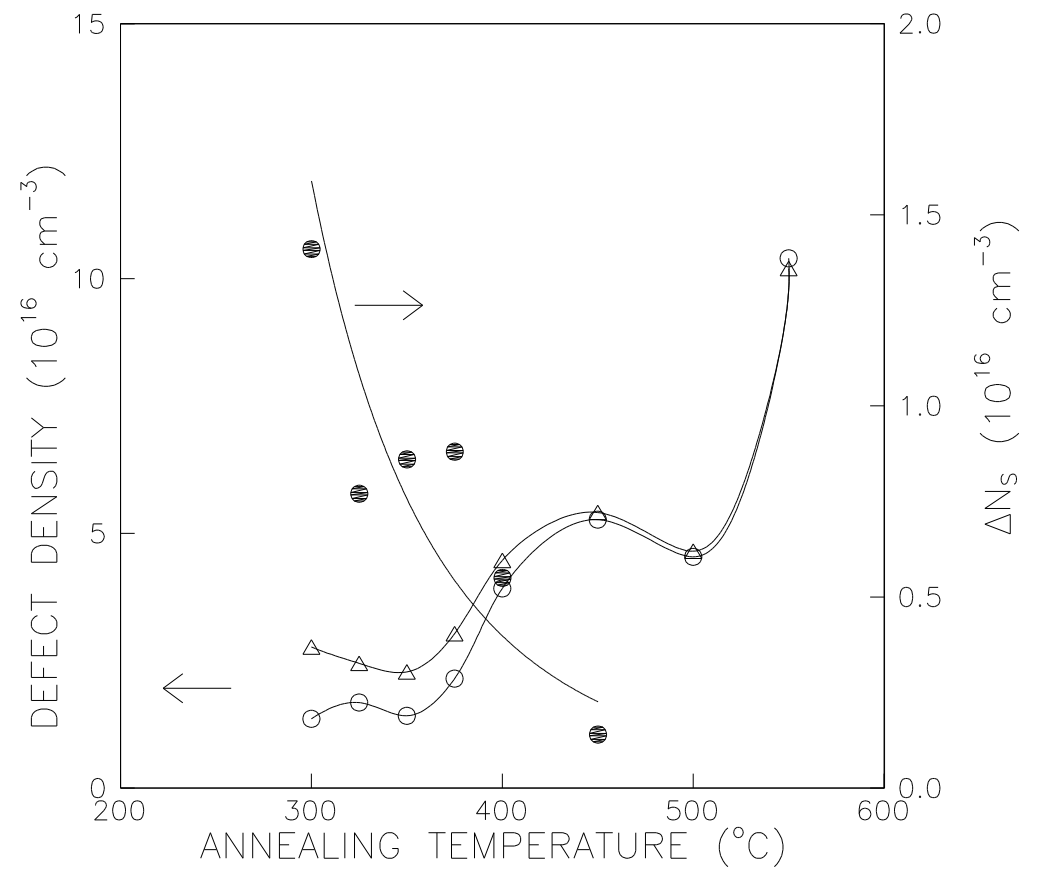




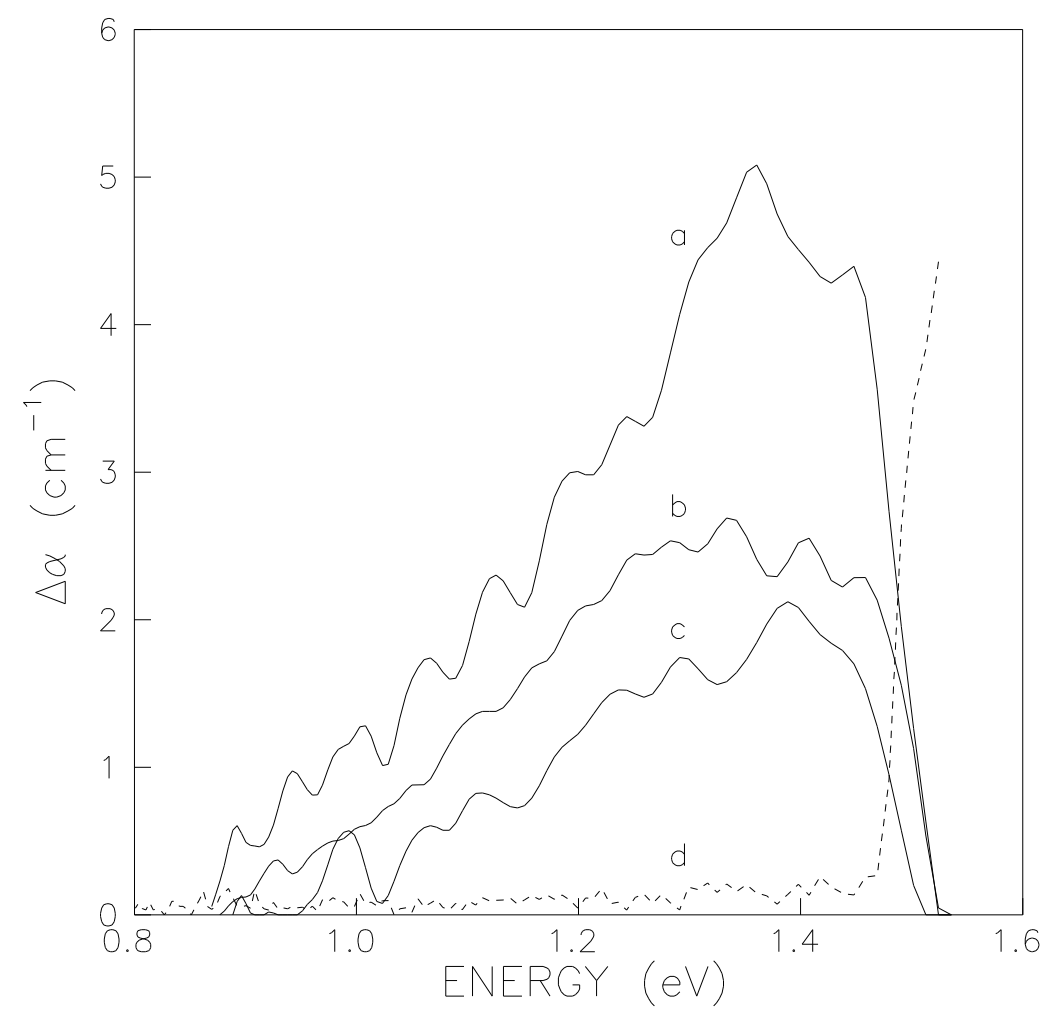




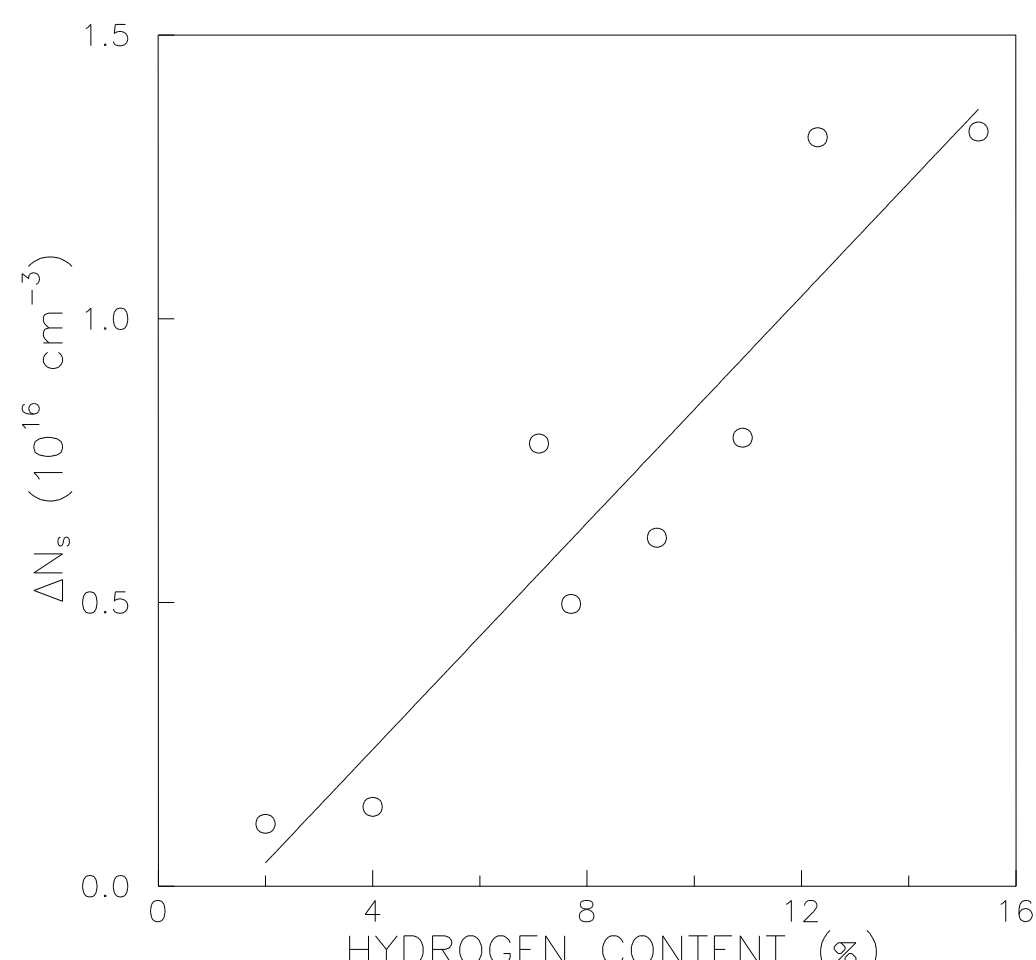

HYDROGEN CONTENT (\%) 